\title{
Willis Eugene Lamb (1913-2008) La passion de la précision
}

Jean-Christophe Pain (jean-christophe.pain@cea.fr)

Laboratoire de Physique Atomique des Plasmas, CEA, DAM, DIF, 91297 Arpajon

En 1947, Willis Eugene Lamb

mesure, avec Robert Retherford,

un écart entre les deux niveaux

de structure fine $2 s_{1 / 2}$ et $2 p_{1 / 2}$

de l'atome d'hydrogène,

alors que l'équation de Dirac

prédisait qu'ils avaient la même

énergie. Cette découverte

de ce que l'on appelle le

"déplacement de Lamb »,

et pour laquelle il reçut le prix

Nobel de physique en 1955,

se révéla décisive pour le

développement de l'électro-

dynamique quantique relativiste,

juste après la Seconde Guerre

mondiale.

Lamb a également contribué

à de nombreux domaines de

la physique quantique, comme

l'éjection d'électrons par les

atomes métastables ou encore

la condensation de Bose-Einstein

et a prédit, en 1939, les transitions

nucléaires qui conduiront,

22 ans plus tard, à la découverte

de l'effet Mössbauer.

\section{Des débuts très prometteurs}

Le physicien américain Willis Eugene Lamb Junior naît le 12 juillet 1913 à Los Angeles. Il suit ses études supérieures à l'université de Berkeley et y soutient, en 1938, sa thèse effectuée sous la direction de Robert Julius Oppenheimer (1904-1967). Il y étudie les propriétés électromagnétiques des noyaux atomiques, puis rejoint l'université Columbia de New York avant d'être nommé, en 1951, professeur à l'université de Stanford. Il s'établit en 1956 à Oxford (Royaume-Uni) et obtient en 1962 la chaire Henry Ford de l'université Yale à New Haven dans le Connecticut. Il achève sa carrière à l'université de l'Arizona, de 1974 à 2002.

Les premiers travaux de Lamb portent sur la capture des neutrons lents par des matériaux hydrogénés ou par des cristaux. En 1939, à l'âge de vingt-six ans, il se distingue par un article théorique dans lequel il prédit des transitions entre niveaux d'énergie des noyaux atomiques qui vaudront, vingt-deux ans plus tard, le prix Nobel de physique à Rudolf Ludwig Mössbauer, pour la mise en évidence expérimentale et l'interprétation de ces transitions. Ce phénomène de fluorescence résonante sans recul des noyaux, connu sous le nom d'effet Mössbauer [1, 2] (voir encadré, p. 29), est parfois appelé effet Lamb-Dicke-Mössbauer. L'effet Lamb-Dicke concerne en fait plus précisément l'émission de rayonnement par un atome ou une molécule, dont le libre parcours moyen est petit devant la longueur d'onde du rayonnement [3, 4]. Il a pour conséquence le rétrécissement des raies par réduction de l'effet Doppler, du fait des collisions électroniques. Lamb aborde également la condensation de Bose-Einstein ou encore l'extraction d'électrons par bombardement ionique d'une surface métallique.

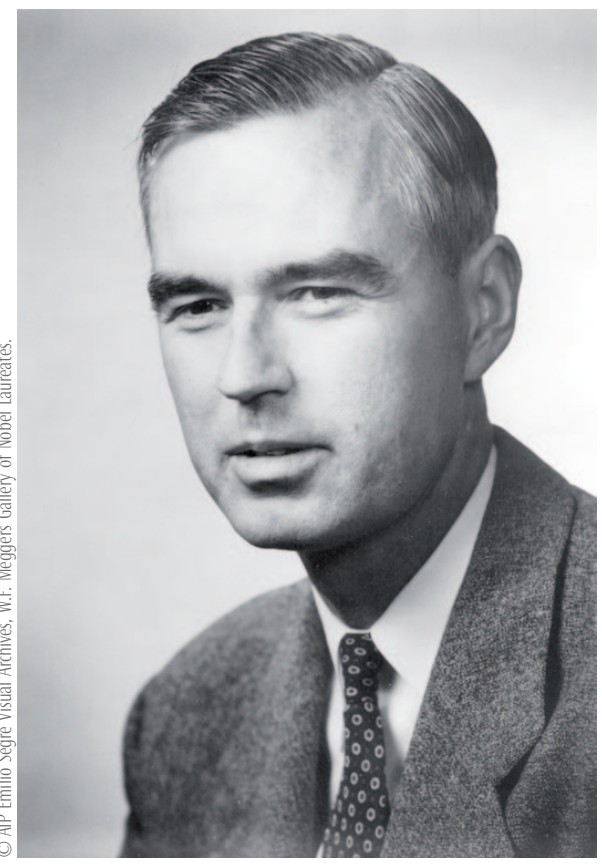

Willis E. Lamb en 1955

Cependant, la guerre oriente les efforts scientifiques aux États-Unis vers les applications militaires. Les recherches sur le radar (et les techniques de construction de tubes à vide) réunissent au laboratoire des rayonnements de Columbia quelques très grands physiciens, dont Isidore Isaac Rabi (prix Nobel de physique en 1944 pour la résonance magnétique nucléaire) et Lamb. L'un de leurs objectifs est de fabriquer des sources de micro-ondes de courtes longueurs d'onde.

En 1939 Lamb épouse Ursula Schaefer, une réfugiée de Berlin contrainte de quitter l'Allemagne pour avoir distribué des pamphlets dénonçant le régime nazi. Être marié à une Allemande à l'aube de la Seconde Guerre mondiale n'est pas sans conséquences pour Lamb ; ce dernier est notamment interdit de plage, car le gouvernement américain redoute qu'il n'entre en contact avec des sous-marins allemands... 


\section{\> \\ Le déplacement de Lamb et l'électrodynamique quantique}

En 1947, Willis Lamb mesure, avec Robert Retherford (1912-1981), un écart entre les deux niveaux de structure fine $2 \mathrm{~s}_{1 / 2}$ et $2 \mathrm{p}_{1 / 2}$ ( $\mathrm{n}=2, \mathrm{j}=1 / 2)$ de l'atome d'hydrogène, qui devaient coïncider d'après l'équation de $\operatorname{Dirac}^{(\mathrm{a})}$. Cette correction est appelée « déplacement de Lamb " ou "Lamb shift" [5]. Lamb et Retherford utilisent des micro-ondes pour stimuler la transition entre les états $2 \mathrm{~s}_{1 / 2}$ et $2 \mathrm{p}_{1 / 2}$ (figs 1 et 2 ). À ces basses fréquences, l'effet Doppler devient négligeable et la différence d'énergie qu'ils mesurent est de l'ordre de $1058 \mathrm{MHz}$ (fig. 3).

C'est Hans Albrecht Bethe qui, à la fin des années 1940, propose la première interprétation théorique du déplacement de Lamb [6]. Son travail pose les fondations de l'électrodynamique quantique moderne, pour laquelle Richard Feynman, Julian Schwinger et Sin-Itiro Tominaga obtiendront le prix Nobel de physique en 1965.

L'électrodynamique quantique (en anglais "Quantum electrodynamics": QED) est une théorie physique conciliant l'électromagnétisme et la mécanique quantique. Dans cette théorie, qualifiée de "bijou de la physique " par Feynman [7], les charges électriques interagissent par l'émission et la réabsorption de photons virtuels, ce qui modifie l'interaction coulombienne entre particules chargées. D'après le principe d'indétermination de Heisenberg, le produit de l'incertitude sur l'énergie par l'incertitude sur le temps est obligatoirement supérieur à une valeur strictement positive (la constante de Planck divisée par $2 \pi$ ). Le vide possède donc de l'énergie, appelée énergie de point zéro. Ainsi, même dans le vide, il existe des champs électromagnétiques associés à l'énergie de point zéro, ce sont les «fluctuations quantiques du vide ». La "renormalisation de la masse de l'électron » consiste à redéfinir cette masse en interaction avec les photons, en y incluant l'auto-interaction résultant de «l'habillage » de l'électron par des photons virtuels [8]. Cette renormalisation entraîne une correction à l'énergie, particulièrement importante pour les niveaux «s ». Par ailleurs, l'équation de Dirac prédit une valeur du rapport gyromagnétique g (rapport entre les moments magnétique et cinétique orbitaux) de l'électron égale à 2 et, lorsqu'on prend en compte l'interaction de l'électron avec le champ électromagnétique quantifié, on trouve que $\mathrm{g}$ differre légèrement de 2 (il vaut en fait 2,002319304386). La renormalisation

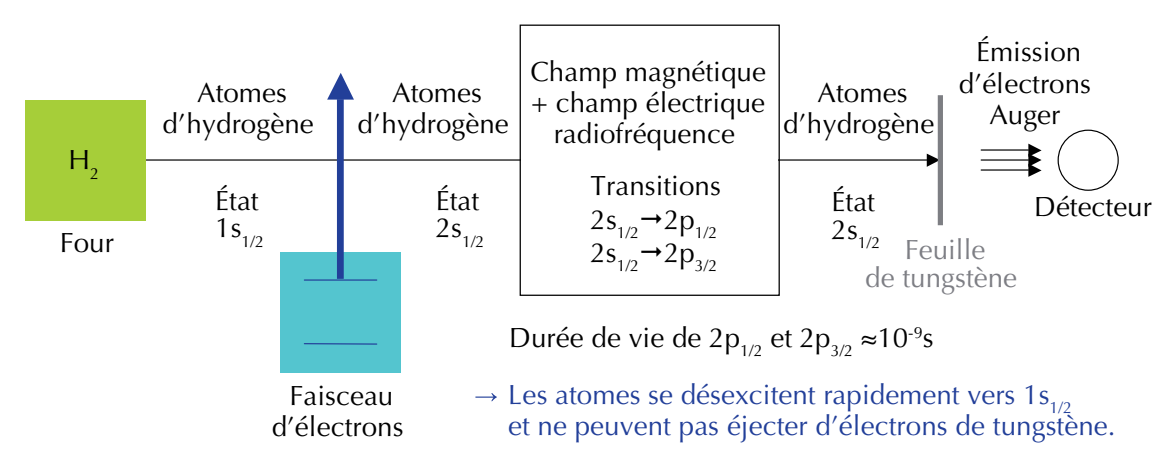

1. Schéma de l'expérience de Lamb et Retherford. Les atomes d'hydrogène, obtenus par dissociation thermique de $\mathrm{H}_{2}$, sont préparés dans l'état $1 s_{1 / 2}$, puis excités dans l'état métastable $2 s_{1 / 2}$ par un faisceau d'électrons. L'application d'un champ magnétique lève la dégénérescence des niveaux par effet Zeeman, et un champ radiofréquence de fréquence adaptée induit des transitions à partir de l'état $2 s_{1 / 2}$ vers les états $2 p_{1 / 2}$ et $2 p_{3 / 2}$. Après avoir traversé la région où règne le rayonnement électromagnétique, les atomes d'hydrogène métastables $2 s_{1 / 2}$ entrent en collision avec une cible de tungstène. Des électrons de la cible sont éjectés et recueillis par un détecteur. Les atomes se trouvant dans les états $2 p_{1 / 2}$ et $2 p_{3 / 2}$ ayant une durée de vie très courte $\left(\sim 10^{-9} s\right)$, se désexcitent très rapidement (avant d'atteindre la feuille de tungstène) vers l'état fondamental $1 s_{1 / 2}$ et ne peuvent pas éjecter des électrons de tungstène, d'où une baisse de courant dans le détecteur observée à la résonance quand on fait varier la fréquence ou le champ magnétique. La variation mesurée par Lamb et Retherford était de l'ordre de $10^{-14}$ ampères !

Effet Zeeman (champ magnétique)

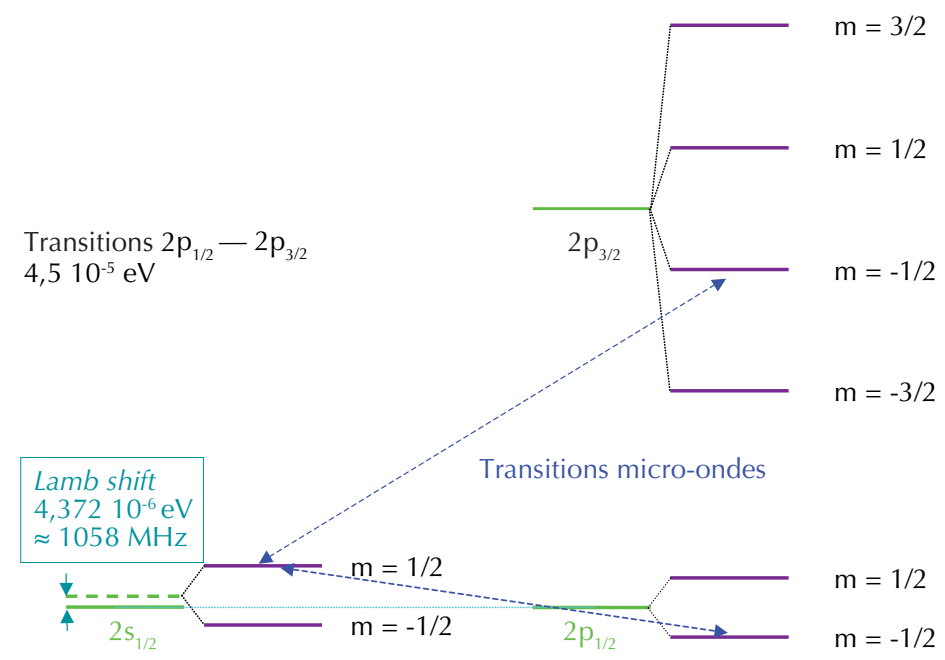

2. C'est l'excitation de la transition entre sous-niveaux Zeeman de $2 s_{1 / 2}$ et de $\left(2 p_{1 / 2}, 2 p_{3 / 2}\right)$ qui a permis à Lamb et Retherford, grâce aux formules de l'effet Zeeman, d'accéder au décalage en énergie de $2 s_{1 / 2}$ (on connait exactement la position relative d'un sous-niveau Zeeman par rapport à son niveau « parent »).
Structure fine

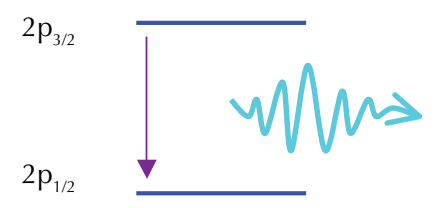

$\begin{aligned} \mathrm{E} & =4,510^{-5} \mathrm{eV} \\ v & =10,9 \mathrm{GHz} \\ \lambda & =2,7 \mathrm{~cm}\end{aligned}$ (couplage spin-orbite)
Lamb shift

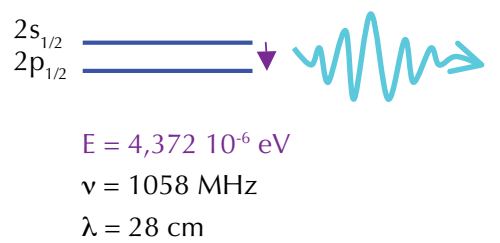

3. Dans l'atome d'hydrogène, le décalage de Lamb est de l'ordre d'un dixième de l'énergie de la transition de structure fine $2 p_{1 / 2}-2 p_{3 / 2}$. Remarque : les modifications d'énergie dues à la structure hyperfine sont inférieures d'un ordre de grandeur au Lamb shift pour les niveaux $2 s_{1 / 2}$ et $2 p_{1 / 2}$. 
de la masse et l'anomalie du moment magnétique sont deux conséquences directes des fluctuations quantiques du vide. L'électron est moins lié au noyau, son énergie de liaison est réduite et l'énergie du niveau $2 \mathrm{~s}_{1 / 2}$ est augmentée de $1085 \mathrm{MHz}$. Un autre phénomène de QED responsable du décalage de Lamb est la "polarisation du vide ". L'équivalence masse-énergie formulée par Einstein implique qu'il est possible de créer des particules massiques dans le vide. Cela se traduit par l'apparition, pendant un temps très court, de paires de particules virtuelles. Des paires virtuelles électron-positron sont créées en permanence et annihilées par le champ coulombien du proton (fig. 4). La charge du proton est donc modifiée par le "nuage " de ces paires virtuelles, et les électrons traversant ce nuage subissent, sur une distance de l'ordre de la longueur d'onde de Compton $^{(b)}$, une attraction plus grande du noyau que ne le prédit le potentiel de Coulomb (comme s'ils étaient "poussés " vers le noyau par les électrons virtuels et attirés vers celui-ci par les positrons virtuels), et sont par conséquent plus fortement liés. Les électrons «s " ont une plus grande probabilité d'être proches du noyau que des électrons " $\mathrm{p}$ » ou « $\mathrm{d}$ ", et sont donc sujets à une plus grande augmentation de leur énergie de liaison. Les calculs montrent que la contribution à la différence d'énergie entre $2 \mathrm{~s}_{1 / 2}$ et $2 \mathrm{p}_{1 / 2}$ due à la polarisation du vide est $-27 \mathrm{MHz}$ (ce décalage va dans le sens opposé de celui imputable aux fluctuations du vide).

La différence entre les mesures expérimentales et les valeurs théoriques est très faible. Lamb obtient le prix Nobel de physique en 1955 pour ses découvertes concernant la structure fine du spectre de l'hydrogène, partagé avec Polykarp Kusch pour la détermination du moment magnétique de l'électron avec une très grande précision. Des calculs récents, incluant tous les effets contribuant au décalage de Lamb, ont donné $1057,833(4) \mathrm{MHz}$, alors que la mesure expérimentale la plus précise est 1057,845(4) $\mathrm{MHz}$ [9].

Des travaux extrêmement précis, réalisés à Garching par Theodor Hänsch et ses collègues [10], ont montré que la fréquence (dans l'ultraviolet) de transition entre les états $1 s_{1 / 2}$ et $2 s_{1 / 2}$ de l'atome d'hydrogène est de 2,446061 $413187103(46) \times 10^{15} \mathrm{~Hz}$.

La connaissance du décalage de Lamb est utile, notamment pour la résonance magnétique nucléaire et l'imagerie associée.

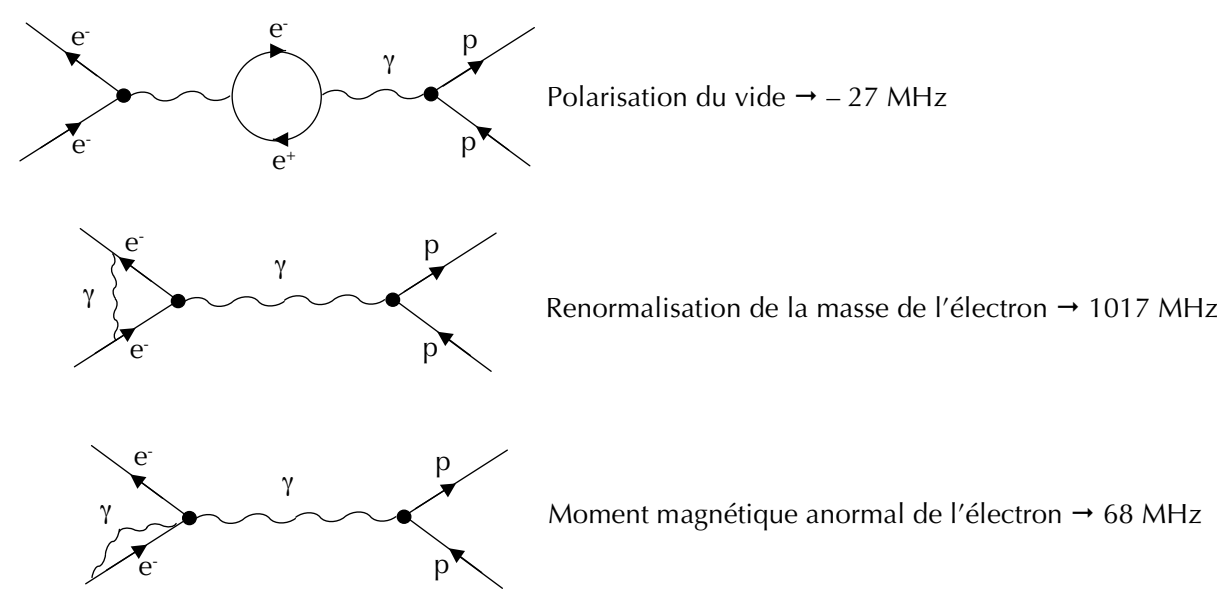

4. Diagrammes de Feynman traduisant les trois processus dominants contribuant au déplacement de Lamb. Ces diagrammes sont des représentations graphiques de différents termes dans le développement en perturbation d'une amplitude de diffusion. En électrodynamique quantique (comme ici), on peut obtenir une excellente approximation de l'amplitude de diffusion à partir de quelques termes de ce développement, correspondant à des diagrammes de Feynman simples. Les particules sont représentées par des lignes, qui peuvent être dessinées de différentes manières en fonction du type de particule représenté. Ici, les lignes droites représentent des électrons (e-) ou des protons (p), les lignes ondulantes des photons virtuels, et le cercle une paire électron-positron virtuelle. La partie inférieure du diagramme représente le passé, et la partie supérieure le futur.

\section{L'effet Mössbauer}

L'effet Mössbauer est lié à l'absorption et à la réémission résonante d'un photon par le noyau d'un atome (transition nucléaire). Les lois de conservation de l'énergie et de l'impulsion imposent une modification de l'énergie des photons réémis dans une direction différente de la direction incidente. Ce décalage en énergie traduit l'effet de recul de l'atome à l'absorption et à l'émission. Cependant, dans un réseau cristallin, l'énergie de recul du noyau est transmise au cristal et la vitesse de recul du noyau est négligeable. Ce dernier est alors susceptible d'émettre ou d'absorber un photon sans recul : c'est l'effet Mössbauer. On a accès ainsi, en absorption et en émission, à une résonance sur la différence d'énergie entre les états fondamental et excité du noyau. Cet effet est utilisé en spectrométrie, afin de déterminer le degré d'oxydation et l'environnement d'éléments chimiques.

\section{Références}

1- R.L. Mössbauer, "Kernresonanz-Fluoreszenz von Gamma-Strahlung in "191r", Z. Physik 151 (1958) 124-143

2• A. Abragam, L'Effet Mössbauer, Gordon and Breach (1964).

3 - W.E. Lamb Jr., "Capture of neutrons by atoms in a crystal lattice", Phys. Rev. 55 (1939) 190.

4 R.H. Dicke, "The Effect of Collisions upon the Doppler Width of Spectral Lines", Phys. Rev. 89 (1953) 472.

5• W.E. Lamb et R. C. Retherford, "Fine structure of the hydrogen atom by a microwave Method", Phys. Rev. 72 (1947) 241-243

6• H.A. Bethe, "The Electromagnetic Shift of Energy Levels", Phys. Rev. 72 (1947) 339-341.

7• R. Feynman, Lumière \& matière : une étrange histoire. Points Sciences 86, Le Seuil (1999).

8- F.A. Reuze, Électrodynamique et optique quantiques, Presses Polytechniques et Universitaires Romandes, Lausanne (2007).

9• M.I. Eides, H. Grotch et V.A. Shelyuto, "Theory of light hydrogenlike atoms", Phys. Rep. 342, (2001) 63-261.

10• T.W. Hänsch, I.S. Shahin et A.L. Schawlow, “0ptical resolution of the Lamb shift in atomic hydrogen by laser saturation spectroscopy", Nature 235 (1972) 63-65.

(a) L'équation de Dirac a été formulée par Paul Dirac en 1928, alors qu'il tentait de transformer l'équation de Schrödinger afin de la rendre compatible avec la relativité restreinte. Cette équation, qui décrit le comportement quantique et relativiste de particules élémentaires de spins demi-entiers, comme les électrons, a permis de prédire l'existence des antiparticules.

(b) La longueur d'onde de Compton peut être considérée comme une limitation fondamentale à la mesure de la position d'une particule, tenant compte de la mécanique quantique et de la relativité restreinte. Elle représente la limite au-dessous de laquelle la théorie quantique des champs - qui permet de décrire la création et l'annihilation de particules - devient importante. 